\title{
Elicitação de Requisitos a partir de Modelos de Processos de Negócio em BPMN: Uma Revisão Sistemática
}

\author{
Alternative Title: Requirements Elicitation from Business Process Model \\ in BPMN: A Systematic Review \\ Aryslene S. Bitencourt* \\ Universidade Federal de Mato \\ Grosso do Sul \\ Faculdade de Computação \\ Caixa Postal 549 - 79070-900 \\ - Campo Grande - MS \\ aryslenebitencourt@hotmail.debora@facom.ufms.br \\ Débora Maria B. Paiva ${ }^{\dagger}$ \\ Universidade Federal de Mato \\ Grosso do Sul \\ Faculdade de Computação \\ Caixa Postal 549 - 79070-900 \\ Campo Grande - MS \\ Maria Istela Cagnin $\ddagger$ \\ Universidade Federal de Mato \\ Grosso do Sul \\ Faculdade de Computação \\ Caixa Postal 549 - 79070-900 \\ - Campo Grande - MS \\ istela@facom.ufms.br
}

\begin{abstract}
RESUMO
A elicitação de requisitos é uma fase complexa da Engenharia de Requisitos, sendo necessários técnicas e métodos específicos para executá-la. Ressalta-se que o sistema de software deve atender plenamente as peculiaridades do negócio da empresa. Assim, com o intuito de elicitar os requisitos adequados para o software apoiar de maneira efetiva o negócio, existem na literatura técnicas e métodos que realizam a extração de requisitos a partir de modelos de processos de negócio, os quais podem ser representados em várias notações, sendo a Business Process Model and Notation (BPMN) a notação padrão atual. Nesse contexto, este trabalho apresenta um revisão sistemática para identificar os trabalhos existentes que realizam a extração de requisitos funcionais e não funcionais tomando como base modelos de processos de negócio na notação BPMN. A partir dos resultados da revisão sistemática observou-se que há poucos trabalhos sobre o tema e que a maioria leva em consideração apenas a extração de requisitos funcionais. Com isso, foi possível delinear pesquisas futuras para contribuir com o avanço nessa área de pesquisa.
\end{abstract}

\section{Palavras-Chave}

Engenharia de Requisitos, Elicitação de Requisitos, Modelos de Processos de Negócio.

\footnotetext{
*Apoio financeiro do CNPq.

${ }^{\dagger}$ Apoio financeiro da Fundect - T.O. no ${ }^{\circ}$ 219/2014 - SIAFEM $n^{\circ} 23963$.

${ }_{\ddagger}$ Apoio financeiro da Fundect - T.O. n $115 / 2014$ - SIAFEM $\mathrm{n}^{\mathrm{o}} 23710$.
}

Permission to make digital or hard copies of all or part of this work for personal or classroom use is granted without fee provided that copies are not made or distributed for profit or commercial advantage and that copies bear this notice and the full citation on the first page. To copy otherwise, to republish, to post on servers or to redistribute to lists, requires prior specific permission and/or a fee.

SBSI 2016, May 17th-20th, 2016, Florianópolis, Santa Catarina, Brazil Copyright SBC 2016.

\begin{abstract}
The requirements elicitation is a complexity phase of the Requirement Engineering, being necessary methods and techniques to execute it. Many researchers emphasized that the software system must fully meet the peculiarities of the company's business. Then, with the intention to elicit rightly the requirements of the software, there are techniques and methods in the literature that perform the requirements extraction from business process models. Such models can be represented in different types of notations, being the $\mathrm{Bu}$ siness Process Model and Notation (BPMN) the standard notation. In this context, this paper describes a systematic review to identify primary studies that perform the extraction of functional and non functional requirements from business process model represented in BPMN notation. From the results obtained through the systematic review, we observed that there are few studies about the subject and most of them take in account only the functional requirements extraction. Thus, it was possible to outline future research to contribute to the advancement in this research area.
\end{abstract}

\section{Categories and Subject Descriptors}

D.2.1 [Software Engineering]: Requirements-elicitation methods

\section{General Terms}

Requirement Engineering

\section{Keywords}

business process model, functional requirement, non functional requirement

\section{INTRODUÇÃO}

Uma das fases iniciais do desenvolvimento de software é a Engenharia de Requisitos [36], sendo considerada uma das mais importantes e complexas. Devido a isso, existem abordagens específicas para apoiar as principais atividades da Engenharia de Requisitos, como elicitação, análise, especificação, validação e gestão de requisitos. A elicitação de requisitos tem como intuito buscar, encontrar, analisar e acom- 
panhar a evolução das necessidades e exigências dos clientes, assim como os conceitos, domínio, tecnologias e limitações que envolvem o sistema de software como um todo [26] [11]. Diante disso, o desenvolvimento do software depende da boa condução da atividade de elicitação de requisitos, pois, caso seja indevidamente realizada, podem surgir requisitos incorretos, incompletos ou conflitantes, podendo levar o projeto ao fracasso [36].

Um bom entendimento do negócio da empresa durante a elicitação de requisitos gera um aumento na qualidade do produto de software. Contudo, a forma com que os requisitos são definidos a partir da elicitação de requisitos com técnicas tradicionais, comumente não reflete o ambiente de negócios [11]. Para contornar esse problema, os profissionais de Engenharia de Software têm notado a importância dos processos do negócio para colaborar na elicitação de requisitos [32] [38]. Os modelos de processos de negócio da organização descrevem o seu comportamento padrão, sendo de grande utilidade para o analista de sistemas, permitindo compreender corretamente o ambiente de negócios e, consequentemente, os requisitos do sistema [39]. Com isso, conduzir a elicitação de requisitos tomando como base modelos de processos de negócio leva a obtenção de requisitos mais precisos e, assim, ao desenvolvimento mais adequado do software para atender as necessidades reais da empresa [8]. Existem várias notações para representar modelos de processos de negócio, sendo a Business Process Model and Notation (BPMN) [28] a notação padrão atual e, portanto, de interesse deste trabalho.

Segundo Xavier [39], existe a necessidade de uma boa comunicação entre analista de negócio e analista de sistema, contudo, a existência de uma lacuna entre os domínios do negócio e o do desenvolvimento de software causa discrepância entre "o que" os usuários finais verbalizam e "o que" o analista de sistema compreende. As organizações enfrentam grande concorrência global, em que o foco é mantido no cliente bem como em tornar os processos de negócios mais ágeis. Assim, para manter vantagem competitiva é necessária a antecipação das mudanças no ambiente de negócios que refletem nos requisitos dos sistemas de software [36]. Dessa forma, é conveniente e necessário manter os requisitos do software alinhados com o negócio da organização.

Para minimizar os problemas supracitados, existem na literatura abordagens que auxiliam a elicitação de requisitos a partir de modelos de processos de negócio, com o objetivo de facilitar a construção e a manutenção de sistemas de software. Diante da importância disso, este trabalho apresenta uma revisão sistemática para identificar as abordagens existentes que apoiam a elicitação de requisitos (funcionais e não funcionais) a partir de modelos de processos de negócio em BPMN. A partir dos resultados obtidos observou-se que há poucos trabalhos nesse contexto e que há carência de abordagens que levam em consideração o levantamento de requisitos não funcionais sob essa perspectiva.

A escrita deste artigo está organizada em mais três seções. $\mathrm{Na}$ Seção 2 são apresentados os principais conceitos e definições sobre modelos de processos de negócio. Na Seção 3 é apresentada a condução da revisão sistemática de acordo com o protocolo proposto por Kitchenham [23]. Na Seção 4 são discutidas sugestões de trabalhos futuros para colaborar com o avanço da linha de pesquisa em questão.

\section{MODELO DE PROCESSOS DE NEGÓ- CIO}

Modelo de processos de negócio representa os processos de negócio de uma empresa e permite a documentação, simulação, compartilhamento, implementação, avaliação e melhoramento contínuo das operações, com o intuito de compreender o funcionamento da organização e os aspectos do seu domínio [19] [24]. O processo de negócio representa o caminho necessário para alcançar os objetivos do negócio. Com isso, surgiu a Gestão de Processos de Negócio, com o intuito de identificar, documentar, desenhar, operar, monitorar e melhorar os processos de negócio a fim de obter resultados alinhados aos objetivos da organização [12].

Para realizar a modelagem de processos de negócio de maneira adequada, é necessário utilizar uma técnica de modelagem, que é dividida em uma linguagem de modelagem e um procedimento de modelagem [21]. Esse último descreve os passos aplicados a uma linguagem de modelagem para criar modelos. Atualmente, a notação Business Process Model and Notation (BPMN) [28] é a linguagem de modelagem de processos de negócio mais utilizada [10] e é de interesse deste trabalho, pois também é a notação atual padrão para a representação de processos de negócio.

A BPMN foi desenvolvida pelo Business Process Management Initiative (BPMI) em 2004 e atualmente é mantida pela Object Management Group (OMG). A versão atual BPMN 2.0.2 foi disponibilizada em dezembro de 2013. Segundo a OMG [28], o principal objetivo da BPMN é prover uma notação que seja facilmente compreensível pelos diferentes tipos de interessados. A notação BPMN fornece um conjunto de elementos gráficos para representar os processos de negócios de uma organização. Para isso, possui um conjunto de elementos, os quais são classificados em cinco categorias básicas: Objetos de Fluxo, Objetos de Dados, Objetos de Conexão, Partições e Artefatos. Existem quatro tipos básicos de diagramas que podem ser representados pelo modelo BPMN: Processo, Coreografia, Colaboração e Conversação, sendo o primeiro o de interesse deste trabalho pois é o diagrama comumente utilizado para documentar processos de negócio.

Na Figura 1 é mostrado um diagrama de processo, que descreve o processo de premiação de um candidato. O ícone superior esquerdo apresenta informação adicional sobre a atividade.

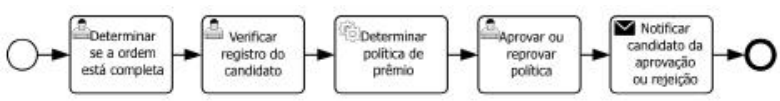

Figura 1: Exemplo de diagrama de processo [28]

\section{REVISÃO SISTEMÁTICA}

A revisão sistemática conduzida e apresentada neste trabalho está dividida em quatro fases: Planejamento, Condução, Extração e Análise dos Dados, de acordo com Kitchenham [23]. O planejamento da revisão sistemática, a seleção dos estudos e a extração de dados foram validados por um especialista na área de Engenharia de Requisitos. Uma breve descrição e os resultados dessas fases no contexto da revisão sistemática conduzida estão apresentados nas seções seguintes. 


\subsection{Planejamento}

Nesta fase é estabelecido o protocolo da revisão sistemática, em que se definem o objetivo, as questões de pesquisa, a estratégia de busca, os critérios de inclusão e de exclusão.

\section{- Objetivo da Pesquisa}

O objetivo da revisão sistemática conduzida neste trabalho é identificar, classificar e analisar estudos que realizam a extração de requisitos funcionais e não funcionais a partir de modelos de processos de negócio representados em diagramas de processos da notação BPMN. Além disso, tem como intuito apresentar uma análise dos estudos selecionados em busca de deficiências e lacunas nessa área de pesquisa para o delineamento e realização de novas pesquisas para contribuir com o avanço do estado da arte.

\section{- Questões de Pesquisa}

As questões de pesquisa definidas buscam efetuar um levantamento das abordagens de extração de requisitos já existentes, levando-se em conta o objetivo de pesquisa supracitado. Portanto, foram definidas duas questões, sendo a QP1 a questão principal e a outra derivação da QP1, conforme apresentadas na Tabela 1 .

Tabela 1: Questões de pesquisa da revisão sistemática

\begin{tabular}{|c|l|}
\hline \multicolumn{2}{|c|}{ Questões de Pesquisa } \\
\hline QP1 & $\begin{array}{l}\text { Como requisitos são extraídos a partir de mo- } \\
\text { delos de processos de negócios? }\end{array}$ \\
\hline QP1.1 & Quais tipos de requisitos são extraídos? \\
\hline
\end{tabular}

\section{- Estratégia de Busca}

Durante a definição da estratégia de busca define-se a string de busca padrão e selecionam-se as bases de buscas. Posteriormente, definem-se as strings de busca específicas para cada base. A construção da string de busca padrão, ilustrada na Tabela 2, foi baseada nas palavras-chave obtidas levando em consideração o objetivo da revisão sistemática. Os termos "functional requirement" e "non functional requirement" foram separados pelo operador "OR", já que os trabalhos relevantes precisam abordar pelo menos um dos tipos de requisitos.

Tabela 2: String de busca padrão da revisão sistemática

\begin{tabular}{|l|l|}
\hline \multicolumn{1}{|c|}{ Palavras-chave } & String de busca padrão \\
\hline 1. Business Process & \\
Model & ("business process model") AND \\
2. Functional & ("functional requirement"OR \\
Requirement & "non functional requirement") \\
3. Non Functional & \\
Requirement & \\
\hline
\end{tabular}

Na Tabela 3 são elencadas as bases de buscas comumente utilizadas em revisões sistemáticas da área de Engenharia de Software. Adicionalmente, foram conduzidas buscas nas principais conferências nacionais e internacionais de Engenharia de Requisitos.
Tabela 3: Bases de busca da revisão sistemática

\begin{tabular}{|l|}
\hline Bases de Busca \\
\hline ACM Digital Library \\
\hline IEEEXplore Digital Library \\
\hline ScienceDirect \\
\hline Springerlink \\
\hline Scopus \\
\hline Web of Science \\
\hline $\begin{array}{l}\text { Biblioteca Digital Brasileira de Teses e Dissertações - } \\
\text { (BDTD) }\end{array}$ \\
\hline Workshop de Engenharia de Requisitos - (WER) \\
\hline Workshop Brasileiro de Gestão de Processos - (WBPM) \\
\hline International Conference on Business Process - (BPM) \\
\hline $\begin{array}{l}\text { International Requirements Engineering Conference - } \\
\text { (RE) }\end{array}$ \\
\hline
\end{tabular}

- Critérios de Inclusão e Exclusão

Os critérios de inclusão e de exclusão foram definidos com o objetivo de encontrar os estudos primários relevantes para a pesquisa. Salienta-se que a definição de critérios permite selecionar, entre os trabalhos retornados, aqueles com maior potencial de responder as questões de pesquisa. Na Tabela 4 são apresentados os critérios definidos.

Tabela 4: Critérios de inclusão e de exclusão da revisão sistemática

\begin{tabular}{|c|c|c|}
\hline Tipo & Id. & Descrição \\
\hline Inclusão & CI1 & $\begin{array}{l}\text { O estudo apresenta uma abordagem } \\
\text { para extrair requisitos a partir de } \\
\text { modelos de processos de negócio em } \\
\text { BPMN }\end{array}$ \\
\hline Inclusão & CI2 & $\begin{array}{l}\text { O estudo descreve uma ou mais ativi- } \\
\text { dades para extrair requisitos de mo- } \\
\text { delos de processos de negócio em } \\
\text { BPMN }\end{array}$ \\
\hline Inclusão & CI3 & $\begin{array}{l}\text { O estudo apresenta uma adaptação } \\
\text { do modelo de processos de negócio em } \\
\text { BPMN para facilitar a extração de re- } \\
\text { quisitos }\end{array}$ \\
\hline Exclusão & CE1 & $\begin{array}{l}\text { O estudo está fora do escopo de elici- } \\
\text { tação de requisitos a partir de mode- } \\
\text { los de processos de negócio em BPMN }\end{array}$ \\
\hline Exclusão & CE2 & O estudo é um tutorial \\
\hline Exclusão & CE3 & $\begin{array}{l}\text { O estudo é resumo de outro trabalho } \\
\text { já selecionado }\end{array}$ \\
\hline Exclusão & $\mathrm{CE} 4$ & $\begin{array}{l}\text { O estudo não está escrito nos idiomais } \\
\text { português ou inglês }\end{array}$ \\
\hline
\end{tabular}

\subsection{Condução}

A fase de condução da revisão sistemática consiste na realização do que foi definido na fase de planejamento. As buscas foram realizadas na segunda quinzena do mês de outubro de 2015. Devido ao grande número de trabalhos retornados das bases de busca, a seleção dos artigos foi dividida em duas etapas descritas a seguir. 
- Primeira Seleção

Foi realizada a busca em cada uma das bases de busca com a aplicação das strings de buscas específicas, bem como nos sites e anais das principais conferências da área de Engenharia de Requisitos. A busca foi realizada tomando como base o título e o abstract dos estudos no período de 2000 até 2015. A partir das buscas realizadas obteve-se o retorno total de 584 artigos.

Desta maneira, para selecionar os trabalhos relevantes ao escopo da revisão sistemática e para eliminar os trabalhos duplicados, foi realizada uma pré-seleção tomando como base o título de cada um dos trabalhos retornados. Para cada artigo identificado como não relevante a partir do título, foi feita também a leitura do resumo para confirmar a exclusão do artigo. Sendo assim, dos 584 artigos retornados foram selecionados para a aplicação dos critérios de inclusão e exclusão 29 artigos, como apresentado na Figura 2. Salienta-se que a seleção dos trabalhos foi validada por outros dois pesquisadores da área.

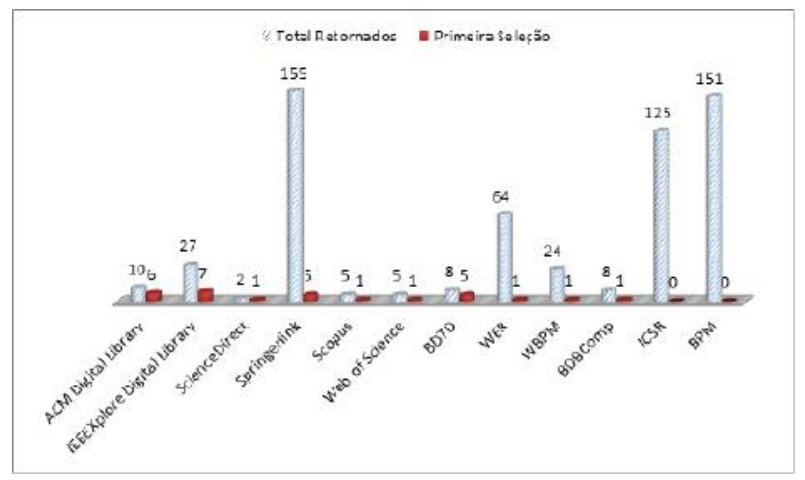

Figura 2: Total de artigos retornados e selecionados por base de busca para aplicação de critérios de inclusão e exclusão

O identificador, a citação bibliográfica, o ano de publicação, o tipo de publicação ([R] evista, $[\mathrm{E}]$ vento, $[\mathrm{D}]$ issertação) e título dos trabalhos obtidos a partir da primeira seleção estão apresentados na Tabela 5. O identificador é formado pela sigla da base de busca que retornou o trabalho mais um número em ordem crescente.

Observou-se que a área de pesquisa abordada pela revisão sistemática é recente, conforme a quantidade de artigos publicados por ano (conforme apresentado na Figura 3), e ainda é imatura, pois poucos dos trabalhos selecionados foram publicados em revista (21\%) e dos 19 artigos publicados em eventos 6 deles (32\%) foram publicados em workshop.

\section{- Segunda Seleção}

Foram aplicados os critérios de inclusão e exclusão nos artigos retornados na primeira seleção. A Tabela 6 apresenta os artigos eliminados por cada critério. Como visto, o critério $\mathrm{CE} 1$, relacionado a notação $\mathrm{BPMN}$, causou a maior eliminação, totalizando $76 \%$ das exclusões, já os critérios CE2 e CE3 totalizaram 7\% dos artigos excluídos. Assim, após a segunda seleção tem-se um total de cinco artigos selecionados para leitura completa: I3E1, WBPM1, BDTD2, BDTD3, BDTD5.

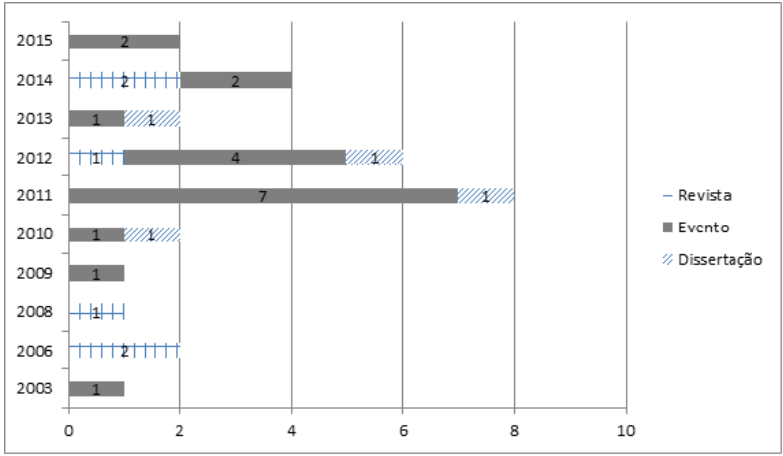

Figura 3: Artigos resultantes da primeira seleção distribuídos no decorrer do tempo

\subsection{Extração e Análise dos Dados}

Para responder as questões de pesquisa definidas na fase de planejamento, foi realizada uma leitura e análise detalhada dos cinco artigos retornados na segunda seleção. A seguir, são elencadas na Tabela 7 as respostas para a questão (QP1 - Como requisitos são extraídos a partir de modelos de processos de negócio?) e subsquestão de pesquisa (QP1.1 - Quais tipos de requisitos são extraídos?) da revisão sistemática, sob a perspectiva de cada trabalho selecionado.

A partir das respostas, observa-se que apenas um trabalho (BDTD3) preocupa-se em extrair requisitos funcionais e não funcionais, além de regras de negócio; dois dos trabalhos selecionados (BDTD5 e I3E1) preocupam-se apenas com a extração de requisitos não funcionais e dois trabalhos (BDTD2 e WBPM1) preocupam-se apenas com a extração de requisitos funcionais. Dos três trabalhos que se preocupam com a extração de requisitos não funcionais com o apoio de heurísticas (BDTD5, BDTD3, I3E1), nenhum deles faz uma avaliação prévia do modelo de processos de negócio para averiguar se o mesmo incorpora aspectos não funcionais a fim de facilitar a aplicação das heurísticas específicas para esse fim.

A abordagem apresentada em BDTD5 proporcionou um melhoramento nas técnicas de elicitação de requisitos não funcionais a partir de modelos de processos de negócio e criou uma extensão para a notação BPMN específica para representá-los, entretanto não explicita os elementos adicionados à notação BPMN e não identifica regras de negócio e requisitos funcionais. Já no trabalho BDTD2 gera-se o documento de requisitos a partir de workshops e análise dos modelos de processos do negócio, contudo não apresenta a metodologia de análise de forma detalhada e também não realiza a extração de requisitos não funcionais.

O trabalho WBPM1 proporciona um aumento na efetividade e na qualidade dos requisitos funcionais a partir do alinhamento organizacional e do negócio da organização, gerando maior precisão nos requisitos visto que os mesmos devem estar em conformidade com o funcionamento do negócio. Porém, não apresenta a metodologia utilizada durante a análise dos modelos de processos para gerar a extração dos requisitos funcionais.

Os trabalhos BDTD3 e I3E1 utilizam heurísticas, as quais apresentam um conjunto de passos e atividades específicas que devem ser praticadas pelo analista de sistema para extrair os requisitos dos modelos de processos de negócio, en- 
Tabela 5: Artigos selecionados para aplicação dos criérios de inclusão e exclusão

\begin{tabular}{|c|c|c|c|c|}
\hline ID & Citação & Ano & Tipo & Título \\
\hline ACM1 & [3] & 2014 & $\mathrm{R}$ & Model checking authorization requirements in business processes \\
\hline ACM2 & [15] & 2012 & $\mathrm{R}$ & $\begin{array}{l}\text { Modeling functional requirements for configurable content- and context-aware dyna- } \\
\text { mic service selection in business process models }\end{array}$ \\
\hline ACM3 & [25] & 2011 & $\mathrm{E}$ & $\begin{array}{l}\text { An agile requirements elicitation approach based on NFRs and business process mo- } \\
\text { dels for micro-businesses }\end{array}$ \\
\hline ACM4 & [11] & 2003 & $\mathrm{E}$ & Utilizing Business Process Models for Requirements Elicitation \\
\hline ACM5 & [4] & 2011 & $\mathrm{E}$ & A BPMN extension for modeling non functional properties of business processes \\
\hline ACM6 & [18] & 2011 & $\mathrm{E}$ & Requirements Elicitation with Business Process Modeling \\
\hline SD1 & [1] & 2006 & $\mathrm{R}$ & Modelling non-functional requirements of business processes \\
\hline I3E1 & [14] & 2011 & $\mathrm{E}$ & Non-functional requirements elicitation from business process models \\
\hline I3E2 & [35] & 2012 & $\mathrm{E}$ & $\begin{array}{l}\text { Evaluating a technique for requirements extraction from business process diagrams } \\
\text { through empirical studies }\end{array}$ \\
\hline I3E3 & [27] & 2012 & $\mathrm{E}$ & $\begin{array}{l}\text { On the expressiveness of business process modeling notations for software require- } \\
\text { ments elicitation }\end{array}$ \\
\hline I3E4 & [20] & 2013 & $\mathrm{E}$ & Software Reuse Based on Business Processes and Requirements \\
\hline I3E5 & [40] & 2012 & $\mathrm{E}$ & From Requirements to Business Processes Development \\
\hline I3E6 & [37] & 2010 & $\mathrm{E}$ & An automatically requirements acquisition method of process business \\
\hline I3E7 & {$[22]$} & 2011 & $\mathrm{E}$ & Considering quality factors for business processes during requirement engineering \\
\hline BDTD1 & {$[9]$} & 2013 & $\overline{\mathrm{D}}$ & $\begin{array}{l}\text { Abordagem para a elicitação de requisitos de software baseada em modelo de processo } \\
\text { de negócio }\end{array}$ \\
\hline BDTD2 & [13] & 2010 & $\mathrm{D}$ & $\begin{array}{l}\text { Uma proposta de abordagem de levantamento de requisitos baseada em modelagem } \\
\text { de processos de negócio }\end{array}$ \\
\hline BDTD3 & [36] & 2012 & $\mathrm{D}$ & $\begin{array}{l}\text { Remo: uma técnica de elicitação de requisitos orientada pela modelagem de processos } \\
\text { de negócios }\end{array}$ \\
\hline BDTD4 & [33] & 2011 & $\mathrm{D}$ & $\begin{array}{l}\text { Sec-MoSC Editor: Modelando composições de serviço com requisitos não funcionais } \\
\text { usando BPMN }\end{array}$ \\
\hline BDTD5 & [39] & 2009 & $\mathrm{E}$ & $\begin{array}{l}\text { Integração de requisitos não-funcionais a processos de negócio: integrando BPMN e } \\
\text { RNF }\end{array}$ \\
\hline WOS1 & [31] & 2014 & E & $\begin{array}{l}\text { Configuring the Variability of Business Process Models Using Non-Functional Re- } \\
\text { quirements }\end{array}$ \\
\hline $\mathrm{SCO} 1$ & [7] & 2011 & $\mathrm{E}$ & $\begin{array}{l}\text { Eliciting goals for business process models with non-functional requirements catalo- } \\
\text { gues }\end{array}$ \\
\hline SPG1 & [16] & 2014 & $\mathrm{R}$ & Literature review of reuse in business process modeling \\
\hline SPG2 & [17] & 2006 & $\mathrm{R}$ & Security requirement analysis of business processes \\
\hline SPG3 & [41] & 2008 & $\mathrm{R}$ & Control case approach to record and model non-functional requirements \\
\hline SPG4 & [29] & 2015 & $\mathrm{E}$ & An Impact Study of Business Process Models for Requirements Elicitation in XP \\
\hline SPG5 & [34] & 2015 & $\mathrm{E}$ & Requirement Elicitation Using Business Process Models \\
\hline WER1 & {$[2]$} & 2014 & $\mathrm{E}$ & $\begin{array}{l}\text { NDR-Tool: Uma Ferramenta de Apoio ao Reuso de Conhecimento em Requisitos Não } \\
\text { Funcionais }\end{array}$ \\
\hline WBPM1 & [6] & 2012 & $\mathrm{E}$ & $\begin{array}{l}\text { Melhorando o Processo Tradicionais de Engenharia de Requisitos sob a Ótica da } \\
\text { Gestão de Processos de Negócio }\end{array}$ \\
\hline BDB1 & [30] & 2011 & $\mathrm{E}$ & $\begin{array}{l}\text { Investigando o uso de conceitos de Linhas de Produto de Software para o reuso de } \\
\text { Modelos de Processos de Negócios }\end{array}$ \\
\hline
\end{tabular}

tretanto no trabalho I3E1 o foco é apenas em requisitos não funcionais, enquanto que no trabalho BDTD3 a preocupação é tanto com a extração de requisitos funcionais como não funcionais, bem como regras de negócio.

Assim, observa-se que o trabalho de BDTD3 apresenta uma abordagem mais completa para a elicitação de requisitos a partir de modelos de processos de negócio em BPMN. Essa abordagem oferece seis heurísticas para identificar requisitos funcionais e duas para identificar requisitos não funcionais, sendo que a identificação das regras de negócio também é tratada juntamente com as heurísticas dos requisitos funcionais. Para a extração dos requisitos e das regras de negócio, é considerado um conjunto de elementos do modelo de processos de negócio, como: operações do processo, condições de decisão contidas no fluxo de sequência dos processos, atividades com dependências de outras operações, atividades com fluxo de interrupções, atividades que derivam mensagens/comunicados, atividades com restrições, atividades com atributos de qualidade). Em cada heurística existe um fluxo de atividades que precisam ser realizadas para executar corretamente a extração. Desta forma, o trabalho proporciona uma metodologia detalhada dos processos necessários para uma boa elicitação de requisitos a partir de modelos de processos de negócio em BPMN. Na próxima seção do artigo são discutidas direções futuras de pesquisa, que tem como intuito propiciar a elicitação de requisitos funcionais e não funcionais com menos esforço e com maior qualidade.

\section{DIREÇÕES FUTURAS E CONSIDERA- ÇÕES FINAIS}

Conforme já discutido, é importante levar em consideração modelos de processos de negócio durante a extração de requisitos funcionais, não funcionais e de regras de negócio a fim de obter requisitos mais completos e consistentes para o desenvolvimento de sistemas de software. Um problema não discutido pelos trabalhos selecionados é o tempo despendido para realizar essa extração.

Com o intuito de reduzir o tempo de elicitação de requisitos a partir de modelos de processos de negócio e aumentar 


\begin{tabular}{|l|l|l|l|l|l|l|}
\hline \multicolumn{1}{|c|}{ Tabela 6: Bases de busca selecionadas } \\
\begin{tabular}{|l|l|l|l|l|l|l|}
\hline Besultados de Busca \\
$\mathbf{1}^{\mathbf{a}}$ Seleção
\end{tabular} & $\mathbf{C E 1}$ & $\mathbf{C E 2}$ & $\mathbf{C E 3}$ & CE4 & Total \\
\hline ACM & 6 & -6 & - & - & - & - \\
\hline I3E & 7 & -5 & -1 & - & - & 1 \\
\hline SD & 1 & -1 & - & - & - & - \\
\hline SPG & 5 & -5 & - & - & - & - \\
\hline SCO & 1 & -1 & - & - & - & - \\
\hline WOS & 1 & - & - & -1 & - & - \\
\hline BDTD & 5 & -2 & - & - & - & 3 \\
\hline WER & 1 & -1 & - & - & - & - \\
\hline WBPM & 1 & - & - & - & - & 1 \\
\hline BDB & 1 & -1 & - & - & - & - \\
\hline Total & $\mathbf{2 9}$ & -22 & $-\mathbf{1}$ & $-\mathbf{1}$ & - & $\mathbf{5}$ \\
\hline
\end{tabular}

Tabela 7: Respostas para a questão de pesquisa da revisão sistemática

\begin{tabular}{|c|c|c|}
\hline ID & QP1 & QP1.1 \\
\hline BDTD5 & $\begin{array}{l}\text { O trabalho extrai requisitos não funcionais a partir de uma extensão do BPMN, deno- } \\
\text { minada framework BPMNFR, cujo intuito é explicitar os requisitos não funcionais em } \\
\text { diagramas de processos de negócio. Tal abordagem consiste nas seguintes atividades: } \\
\text { Construir o Diagrama de Processos de Negócios (BPD); Inserir rótulos nas atividades } \\
\text { do BPD para identificar os requisitos não funcionais; Criar catálogos dos requisitos } \\
\text { não funcionais; e Inserir as operacionalizações no BPD. }\end{array}$ & $\begin{array}{l}\text { Requisito não } \\
\text { funcional }\end{array}$ \\
\hline BDTD2 & $\begin{array}{l}\text { Uma abordagem de elicitação de requisitos baseada em modelos de processos de ne- } \\
\text { gócio que envolve diretamente os usuários, realizando workshops para o levantamento } \\
\text { de requisitos de maneira incremental a partir da análise e discussão dos modelos de } \\
\text { processos de negócio com os usuários. Após o levantamento de requisitos, é feita a } \\
\text { especificação dos mesmos em um documento de requisitos. }\end{array}$ & $\begin{array}{l}\text { Requisito fun- } \\
\text { cional }\end{array}$ \\
\hline BDTD3 & $\begin{array}{l}\text { O trabalho utiliza um conjunto de heurísticas para o analista de requisitos extrair } \\
\text { requisitos funcionais, não funcionais e regras de negócio. Essas heurísticas extraem os } \\
\text { requisitos e as regras de negócio a partir das atividades dos diagramas de processos de } \\
\text { negócios. Para isso, as heurísticas fornecem instruções que guiam o analista durante } \\
\text { a elicitação. }\end{array}$ & $\begin{array}{l}\text { Requisitos } \\
\text { funcional e } \\
\text { não funcional }\end{array}$ \\
\hline WBPM1 & $\begin{array}{l}\text { Esse trabalho possibilita introduzir princípios de Gestão de Processos de Negócio } \\
\text { (BPM) durante o processo de extração de requisitos, com o intuito de obter requisitos } \\
\text { mais consistentes com a realidade atual da organização. Primeiramente, elabora-se o } \\
\text { modelo de negócio "As Is" como ponto de partida. Em seguida, o processo desejado } \\
\text { é modelado, obtendo-se o modelo "To Be". Visando o alinhamento dos requisitos } \\
\text { ao negócio foram adicionadas duas atividades na abordagem proposta: alinhamento } \\
\text { organizacional e alinhamento de negócios durante a análise do modelo de processos } \\
\text { de negócios. Porém, o trabalho não fornece detalhes do processo de análise do modelo } \\
\text { de negócio para a extração dos requisitos funcionais. }\end{array}$ & $\begin{array}{l}\text { Requisito fun- } \\
\text { cional }\end{array}$ \\
\hline I3E1 & $\begin{array}{l}\text { Neste trabalho é apresentada uma abordagem de elicitação de requisitos não funcio- } \\
\text { nais por meio de heurísticas sistemáticas que precisam ser seguidas pelo analista de } \\
\text { sistema para obter requisitos consistentes. }\end{array}$ & $\begin{array}{l}\text { Requisito não } \\
\text { funcional }\end{array}$ \\
\hline
\end{tabular}

a qualidade dos requisitos tanto do ponto de vista de sua completude quanto do ponto de vista do seu alinhamento com o negócio, propõe-se o uso de Linhas de Processos de Negócio (LPN) com requisitos associados.

O conceito LPN foi criado em 2006 a partir do conceito Linha de Produto de Software (LPS), adaptado para o contexto de processos de negócio, a fim de gerenciar um conjunto de processos de negócio similares ao invés de apenas um único. Uma LPN é capaz de levantar tanto os componentes invariantes quanto variantes, a fim de capturar as variabilidades do processo e promover a reutilização e a flexibilidade necessária em um ambiente de negócio em constante mudança [5]. Uma LPN busca gerenciar um conjunto de comunalidades, que são as partes comuns do domínio do negócio; um conjunto de variabilidades, que podem ser selecionadas para adequar o processo de destino; e um conjunto de regras, que explicitam a tarefa de tomada de decisão para realizar a composição flexível de ativos de processos de negócio [5].

Assim, os requisitos funcionais, não funcionais e as regras de negócio associados a uma determinada LPN possibilitam que a extração desses seja realizada apenas quando a linha for criada ou evoluída. Com isso, quando a instanciação da LPN for realizada, além de reutilizar os processos de negócio do domínio da linha é opcionalmente possível reutilizar os requisitos (funcionais, não funcionais e regras de negócio) associados, reduzindo o tempo de elicitação de requisitos. Assim, a medida que os requisitos são reutilizados os mes- 
mos são testados e aprimorados, de forma que em outras instanciações da LPN os mesmos tenham maior qualidade. Devido a complexidade e a dinamicidade dos negócios, é imprescindível apoio computacional para apoiar a criação, instanciação e evolução de LPNs com requisitos associados.

Dessa forma, este trabalho apresentou uma revisão sistemática para levantar os estudos primários que tratam da extração de requisitos funcionais e não funcionais a partir de modelos de processos de negócio elaborados em BPMN. Apesar da relevância desse tema, poucos trabalhos foram encontrados com a revisão sistemática realizada. Com isso, foi possível observar que a área de pesquisa observada é recente e, consequentemente, ainda imatura abrindo diversas possibilidades para pesquisas futuras, como as citadas a seguir: i) adaptação de abordagens existentes de criação, instanciação e evolução de LPNs para permitir a associação de requisitos (funcionais, não funcionais e regras de negócio) a LPNs com o intuito de reduzir o tempo para a obtenção de requisitos e aumentar a qualidade dos mesmos; ii) desenvolvimento de ferramentas computacionais para apoiar tais abordagens; e iii) definição de diretrizes para avaliar se é possível ou não extrair requisitos não funcionais a partir de um determinado modelo de processo de negócio ou LPN.

\section{REFERÊNCIAS}

[1] F. Aburub, M. Odeh, and I. Beeson. Modelling non-functional requirements of business processes. Information Software Technology, 49(11-12):1162-1171, 2007.

[2] A. L. Araújo, L. M. Cysneiros, and V. M. B. Werneck. Ndr-tool: Uma ferramenta de apoio ao reuso de conhecimento em requisitos não funcionais. In Workshop de Engenharia de Software. WER, 2014.

[3] A. Armando and S. E. Ponta. Model checking authorization requirements in business processes. Computers $\& 3$ Security, 40(0):1 - 22, 2014.

[4] P. Bocciarelli and A. D'Ambrogio. A BPMN extension for modeling non functional properties of business processes. In 2011 Symposium on Theory of Modeling 85 Simulation: DEVS Integrative MES Symposium, TMS-DEVS'11, page 160-168, San Diego and CA and USA, 2011. Society for Computer Simulation International.

[5] N. Boffoli, D. Caivano, D. Castelluccia, and G. Visaggio. Business process lines and decision tables driving flexibility by selection. In T. Gschwind, F. D. Paoli, V. Gruhn, and M. Book, editors, Software Composition, volume 7306 of Lecture Notes in Computer Science, pages 178-193. Springer, 2012.

[6] E. C. S. Cardoso, J. P. A. Almeida, G. Guizzardi, and R. S. S. Guizzardi. Eliciting goals for business process models with non-functional requirements catalogues. In T. A. Halpin, J. Krogstie, S. Nurcan, E. Proper, R. Schmidt, P. Soffer, and R. Ukor, editors, $B M M D S / E M M S A D$, volume 29 of Lecture Notes in Business Information Processing, pages 33-45. Springer, 2009.

[7] J. H. d. M. Cardoso, A. A. O. Oliveira, and F. Alencar. Melhorando processos tradicionais de engenharia de requisitos sob a ótica da gestão de processos de negócio. In Workshop de Gestão de Processos de Negócio. EBPM, 2012.
[8] E. A. Carvalho, T. Escovedo, and R. N. Melo. Using business processes in system requirements definition. In V. Werneck and K. Breitman, editors, $S E W$, pages 125-130. IEEE, 2009.

[9] M. A. Chiarello. Abordagem para a elicitação de requisitos de software baseada em modelo de processo de negócio. Master's thesis, Universidade Tecnológica Federal do Paraná - Departamento Acadêmico de Informática, Curitiba, 2013.

[10] M. Chinosi and A. Trombetta. BPMN: An introduction to the standard. Computer Standards Interfaces, 34:124-134, 2012.

[11] O. Demirors, C. Gencel, and A. Tarhan. Utilizing business process models for requirements elicitation. In EUROMICRO, pages 409-412. IEEE, 2003.

[12] H.-E. Eriksson and M. Penker. Business Modeling With UML: Business Patterns at Work. Wiley, 1 edition, 2000.

[13] T. M. M. Farias. Uma proposta de abordagem de levantamento de requisitos baseada em modelagem de processos de negócio. Master's thesis, Universidade Federal de Pernambuco - Centro de Informática, Recife, 2010.

[14] A. F. J. Franco. Non-functional requirements elicitation from business process models. In $R C I S$ pages 1-7. IEEE, 2011.

[15] A. Frece and M. B. Juric. Modeling functional requirements for configurable content- and context-aware dynamic service selection in business process models. J. Vis. Lang. Comput., 23(4):223-247, 2012.

[16] N. Z. Haddar, L. Makni, and H. Ben-Abdallah. Literature review of reuse in business process modeling. Software and System Modeling, 13(3):975-989, 2014.

[17] P. Herrmann and G. Herrmann. Security requirement analysis of business processes. Electronic Commerce Research, 6(3-4):305-335, 2006.

[18] L. B. Hvatum. Requirements elicitation with business process modeling. In Pattern Languages of Programs Conference (PLOP), 2014.

[19] N. M. Jossutis. SOA na prática: A arte da modelagem de sistemas distribuídos. In Rio de Janeiro: Alta Books, 2008.

[20] H. Kaindl. Software reuse based on business processes and requirements. In P. Muenchaisri and G. Rothermel, editors, APSEC (2), pages 85-86. IEEE, 2013.

[21] D. Karagiannis and H. Kühn. Metamodelling platforms. Lecture Notes in Computer Science, pages 182-182, 2002.

[22] Z. Kedad and P. Loucopoulos. Considering quality factors for business processes during requirement engineering. In $R C I S$, pages 1-9. IEEE, 2011.

[23] B. Kitchenham and S. Charters. Guidelines for performing systematic literature reviews in software engineering. Technical Report EBSE 2007-001, Keele University and Durham University Joint Report, 2007.

[24] S. Ladeira, R. Penteado, R. Braga, and M. Cagnin. Reutilização de modelagem de negócios baseada em visões - um estudo de caso. In XXII Simpósio 
Brasileiro de Engenharia de Software, pages 140-155. SBES, 2008.

[25] R. Macasaet, L. Chung, J. L. Garrido, M. Noguera, and M. L. Rodríguez. An agile requirements elicitation approach based on nfrs and business process models for micro-businesses. In 12th International Conference on Product Focused Software Development and Process Improvement, Profes '11, pages 50-56, New York, NY, USA, 2011. ACM.

[26] L. E. G. Martins and B. M. Daltrini. Organizando o processo de elicitação de requisitos utilizando o conceito de atividade. In WER, pages 297-317, 2001.

[27] C. Monsalve, A. April, and A. Abran. On the expressiveness of business process modeling notations for software requirements elicitation. In IECON 2012 38th Annual Conference on IEEE Industrial Electronics Society, pages 3132-3137, Oct 2012.

[28] OMG. Business process model and notation (BPMN). Object Management Group, formal/2011-01-03, 2011.

[29] H. Ordoñez, A. F. E. Villada, D. L. V. Vanegas, C. Cobos, A. Ordóñez, and R. Segovia. An impact study of business process models for requirements elicitation in XP. In Computational Science and Its Applications - ICCSA 2015 - 15th International Conference, Banff, AB, Canada, June 22-25, 2015, Proceedings, Part I, pages 298-312, 2015.

[30] E. C. Santana, C. Maciel, and M. A. S. Turine. Investigando o uso de conceitos de linhas de produto de software para o reúso de modelos de processos de negócio. In Simpósio Brasileiro de Sistemas de Informação. SBSI, 2011.

[31] E. Santos, J. Pimentel, J. Castro, J. Sánchez, and O. Pastor. Configuring the variability of business process models using non-functional requirements. In I. Bider, T. A. Halpin, J. Krogstie, S. Nurcan, E. Proper, R. Schmidt, and R. Ukor, editors, $B M M D S / E M M S A D$, volume 50 of Lecture Notes in Business Information Processing, pages 274-286. Springer, 2010.

[32] I. Sommerville. Software Engineering. São Paulo: Addison-Wesley, 8 edition, 2006.

[33] A. R. S. S. Souza. Sec-mosc editor: Modelando composições de serviço com requisitos não funcionais usando BPMN. Master's thesis, Universidade Federal de Pernambuco - Centro de Informática, Recife, 2011.

[34] S. Valvas and F. Milani. Requirement elicitation using business process models. In Perspectives in Business Informatics Research - 14th International Conference, BIR 2015, Tartu, Estonia, August 26-28, 2015, Proceedings, pages 67-81, 2015.

[35] S. Vieira, D. Viana, R. do Nascimento, and T. Conte. Evaluating a technique for requirements extraction from business process diagrams through empirical studies. In XXXVIII Conferencia Latinoamericana en Informatica, pages 1-10, Oct 2012.

[36] S. R. C. Vieira. REMO - uma técnica de elicitação de requisitos orientada pela modelagem de processos de negócios. Master's thesis, Universidade Federal do Amazonas - Instituto de Computação, Manaus, 2012.

[37] Y.-h. Wang, G.-s. Yin, L. He, and J.-f. Yu. An automatically requirements acquisition method of process business. In Industrial Mechatronics and
Automation (ICIMA), 2010 2nd International Conference on, volume 1, pages 502-505, May 2010.

[38] R. S. Wazlawick. Análise e design orientados a objetos para sistemas de informação: Modelagem com UML, OCL e IFMS. Elsevier, 2015.

[39] L. Xavier. Integração de requisitos não-funcionais a processos de negócio: integrando BPMN e RNF. Master's thesis, Universidade Federal de Pernambuco Centro de Informática, Recife, 2019.

[40] H. Zhang, H. Zou, F. Yang, and R. Lin. From requirements to business processes development. In $I X$ International Conference on Services Computing, Honolulu, HI, USA, June 24-29, 2012, pages 672-673, 2012.

[41] J. Zou and C. J. Pavlovski. Control case approach to record and model non-functional requirements. Inf. Syst. E-Business Management, 6(1):49-67, 2008. 